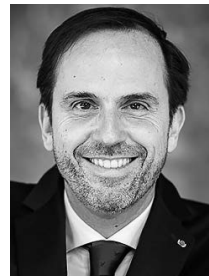

\title{
ES \\ Understanding of pathophysiology and evolution of treatment for CRSwNP
}

\author{
Prof Dr Peter Hellings, MD, PhD
}

Otorhinolaryngology, University of Leuven, University of Ghent, University of Amsterdam, Chair of EUFOREA

Chronic rhinosinusitis (CRS) is a heterogeneous disease characterized by inflammation of the nose and paranasal sinuses with symptoms including nasal blockage/obstruction/congestion, nasal discharge, facial pain/pressure, and reduction or loss of smell for $\geq 12$ weeks.

Classically, CRS is divided into a phenotype with and without nasal polyps (CRSwNP and CRSsNP, respectively). Recently, not only phenotype but also endotype of CRS has been in the spotlight and CRSwNP is associated with a Type 2 inflammatory signature in most cases. Recent findings reveal that part of CRSsNP patients demonstrated type2 inflammation as well.

Type 2 inflammation is characterized by cytokines such as IL-4, IL-5, IL-13, and IL-31 which are often produced by the chronic dysregulation of innate and adaptive immune systems.

Latest research of CRS demonstrated that patients with a pure or mixed type 2 endotype tend to be much more resistant to existing therapies, exhibiting a high recurrence rate when compared with pure type 1 or 3 endotypes.

Promisingly, biologic therapy has now become available that target specific aspects of type 2 inflammation.

EUFOREA (www.euforea.eu) proposed the practical algorithm to define type 2 inflammation in CRSwNP in daily clinical practice and discussed regarding the patient criteria for the selection of biologics and monitoring of its effectiveness based on evidence.

In this presentation, we present latest consensus on definition including pathophysiology for CRSwNP and discus implications for treatment focus on Type2 inflammation.

\section{Curriculum vitae}

Peter Hellings, MD, PhD

Professor of Otorhinolaryngology at the Catholic university of Leuven in Leuven, Belgium; Clinic Head at the Department of Otorhinolaryngology at the University Hospitals of Leuven; and Full Professor at the Academic Medical Center of the University of Amsterdam in the Netherlands, and Guest Professor at the University of Ghent, Belgium.

Professor Hellings' research has led to novel insights into the pathophysiology of allergy and chronic airway inflammation, with over 300 scientific publications in top journals in the field of allergology, rhinology and respiratory care.

Professor Hellings is also the founder of the European Forum for Research and Education in Allergy and Airway Disease, an international nonprofit involving a consortium of European key opinion leaders aiming at the implementation of optimal care for chronic respiratory diseases. He is also founder and director of the European Rhinoplasty Course, and member of European and American guideline committees. 